\title{
Functionally orientated/systemic approach to orthodontic treatment of severe class II division 1 malocclusions: an adapted device, the FABP
}

\author{
J.-L. Raymond \\ Expert qualified in DFO (public practice)
}

\begin{abstract}
The orthodontic treatment of severe class II division 1 malocclusions is often difficult, which leads some specialists to offer a surgical correction of the overjet. Treatment is made complex by the value of the horizontal overlap as much as the " depth " of deep bite that is very often present alongside the malocclusion. This is why we are offering a treatment protocol including a FABP (Fixed Anterior Bite Plate) which will allow, if the patient cooperates, to correct the anatomic anomaly while concurrently establishing new masticatory cycles in order to stabilize and preserve the results obtained. It is this systemic approach of treatment that is the focus of this article.
\end{abstract}

\section{KEYWORDS}

Skeletal class II malocclusions, mandibular movements, occlusal plane, incisal bite plane

Ideally, severe class II/1 cases should be corrected in either temporary or mixed dentition ${ }^{7}$ because early normalization of arch ratios promotes harmonious facial growth and development. Unfortunately, for various reasons (screening, inappropriate or poorly worn appliances, treatment refusal, lack of cooperation from patients or their parents, etc.), it is relatively common that early correction is not done, which forces the clinician to deal with these severe class II/1 cases in adolescents or, in other words, at the orthodontic age.

The major difficulty in orthodontic treatment is to completely reduce the overjet and overbite independently of the etiopathogenesis of dysmorphosis ${ }^{7,15}$ to create favorable anatomical conditions for the establishment of a physiological mastication, which is likely to determine the result ${ }^{14}$. This last point constitutes the originality of the systemic approach of the treatment that we propose. Establishing anatomically normal arch ratios is not sufficient if they are not fitted, concomitantly, with a functional anterior guide, that is to say adapted to a new masticatory functional scheme on which the stability of the correction depends. It is this approach that we describe in this article.

Address for correspondence:

Jean-Louis Raymond - Medical Center - 8 rue du Docteur René-Marques -

66250 Saint-Laurent-de-la-Salanque

E-mail: raymond.jean-louis@wanadoo.fr

Article received: 08-01-2018. Accepted for publication: 14-02-2018.

This is an Open Access article distributed under the terms of the Creative Commons Attribution License (http://creativecommons.org/licenses/by/4.0), which permits unrestricted use, distribution, and reproduction in any medium, provided the original work is properly cited. 


\section{DEFINITION OF SYSTEMIC ORTHODONTICS}

Systemic orthodontics differs from "contemporary" orthodontics in that it takes into account the masticatory system as a whole (hence its name); in other words, it considers the links between arch morphology and mastication. What we call "contemporary orthodontics" is an orthodontic treatment that focuses primarily on the esthetic alignment of the teeth-for a beautiful smile-often ignoring mastication $^{9}$ and its morphogenetic influence ${ }^{3-6,10,12,14,16}$.

Systemic orthodontics is therefore at the heart of any therapeutic project involving masticatory rehabilitation, whose architectural role is, in our opinion, essential ${ }^{12,14}$. The alignment of the teeth is not considered as an end in itself but as a means to achieve masticatory rehabilitation, which constitutes the fundamental objective of the treatment since it conditions its durability.

The employed treatment methods can be of any type and adapted to any malocclusion, provided that they make it possible to achieve the fixed functional objective, and among other things, conventional multi-attachment devices can often be used in combination with specific auxiliary devices.

\section{SYSTEMIC APPROACH OF SEVERE CLASS I/1 CASES}

\section{Morphological description of severe class II cases}

Concerning the complete description of the various dysmorphoses grouped under the term class $11 / 1$, we refer to more detailed articles $2,7,15$.

Here we simply describe the aspect of occlusal relations for which the therapeutic protocol proposed in this article has been satisfactory.

Class $1 / / 1$ is most often a skeletal anomaly of the mandibular retrognathia type, but can also sometimes be of the maxillary protrusion type. It is a relatively frequent malocclusion and may be a real therapeutic challenge for the orthodontist.

This is probably the reason why some practitioners today do not hesitate to resort more frequently to mandibular advancement surgery. However, it is sometimes possible to correct the severe class II/1 malocclusion with orthodontic treatment using a simpler systemic approach, i.e., by changing the orientation of the occlusal plane and the resulting mandibular kinematics.

In class II/1 cases, the degree of the overjet characterizes the severity of the malocclusion. Although it may be caused by various different factors, this incisal overjet, together with the associated overbite, constitutes a major occlusal obstacle to long-term correction, regardless of the nature of the dysmorphosis.

This overjet is usually $>7-8 \mathrm{~mm}$ and can reach $15-16 \mathrm{~mm}$. The shift between the two arches is the result of a skeletal offset and also, in part, alveolar abnormalities resulting from functional 
disorders that accompany these dysmorphoses (atypical deglutition, lingual and/or labial interposition, etc.).

These alveolar abnormalities are most often of the upper incisal proalveolia type associated either with proalveolia of the lower incisors-possibly related to abnormal lingual pressureor, conversely, with a lingual version of the lower incisors related to pressure exerted on the lower lip.

Due to the skeletal offset between the two arches, a strong overbite often accompanies the pronounced overjet in severe class $11 / 1$ cases. It is the consequence of the absence of contacts-in maximal intercuspation in occlusion (MIO) as well as in functionbetween the upper and lower incisors. While it is often the result of a vertical excess of the upper incisors, there are cases in which the lower incisors are also responsible for the overbite.

\section{Joint (occlusion) and functional aspect of severe class II division 1 cases}

\section{Frontal kinematics}

The exploration of the mandibular kinematics is a reflection of the masticatory cycles ${ }^{12,14}$; it is therefore often performed during clinical examinations, especially if one has a systemic approach to orthodontics. For us, the aspect of the mandibular kinematics characterizes both class II/1 dysmorphosis and alveolar dental anomalies. Moreover, the change in mandibular kinematics is one of the treatment objectives in the same manner as anatomical corrections, of which physiological mastication is the functional outcome. The exploration of correctly executed mandibular lateral movements shows an almost exclusively frontal kinematic profile, i.e., without a propulsive component. It shows the trajectory of the lower incisors and their distance from the upper incisal block.

As for the lower canines, their mesial side is articulated with the distal side of the upper canines. In a very large number of cases, this kinematics is symmetrical on the right and on the left, and its vertical component is relatively weak, so that we observe very little lowering of the mandible during the exploration of the induction movements.

\section{Occlusal plane orientation}

This mandibular kinematics frequently observed in severe class $11 / 1$ is essentially due to the spatial orientation of the occlusal plane, which presents an abnormal orientation, characteristic of this type of malocclusion, namely diverging forward with the Camper plane (Fig. 1).

From the outset, it should be noted that, in most cases, the orientation of

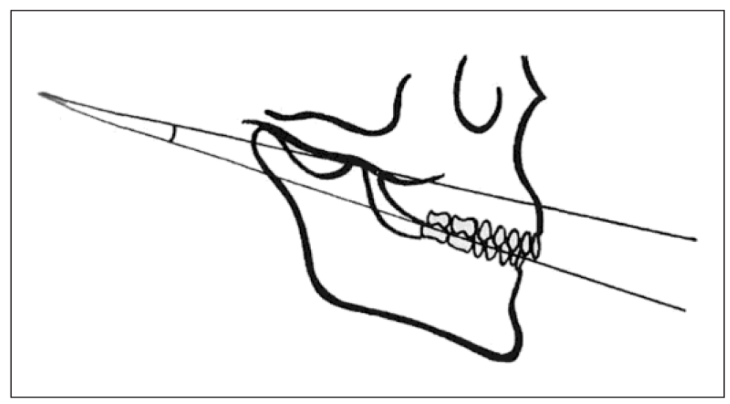

Figure 1

Schematic of the occlusal plane orientation, diverging with the Camper plan in Class II. 
the occlusal plane is not identifiable on profile teleradiographies because of its small inclination; the angle is low in degrees but has severe repercussions on arch articulation and development.

Moreover, as we have said before, the linear representation of the occlusal plane in cephalometry does not reflect its "occlusal reality" - in the form of a twisted ribbon-which is a real source of confusion. In short, cephalometry is not the tool to measure occlusal plane orientation!

Without being able to visualize and measure the inclination of the occlusal plane, we still have the resource, although sometimes delicate, to appreciate the influence of its spatial orientation on mandibular kinematics. This also applies to other malocclusions such as Class $1{ }^{113}$. Therefore, for severe class II/1 cases, we would observe a frontal kinematics devoid of a propulsive component as a consequence of the divergence of the occlusal plane with the Camper plane. long-term correction of most severe class II/I in adolescents.

\section{Overjet and overbite correction}

The correction of an overjet and overbite is done simultaneously through the use of an auxiliary called the fixed anterior bite plate (FABP, described earlier) in conjunction with the use of Class II elastics. We will return to the terms of use and the operation of the device when describing it. The correction and even hypercorrection-of overbites is an essential prerequisite for obtaining a change in mastication (mandibular kinematics) that occurs with the reorientation of the occlusal plane.

\section{Correction of occlusal plane orientation}

As stated above, correction of occlusal plane orientation is necessary for the long-term stability of morphological correction.

Indeed, it is useless to change the shape of the arches and correct the overjet, if we do not set up functions adapted to this new morphology at the same time, i.e., if we do not rehabilitate the unilateral physiological mastication $^{3,7,12,14}$.

But why attach so much importance to This goal can be summarized by a the mandibular kinematics of these malocclusions? Because, in general, kinematics is a reflection of the threedimensional relationships of the arches, and these relationships must logically change with orthodontic treatment. Specifically, it should lead to a new kinematics that involves the incisors if one wants to increase the chances of the stability of long-term correction.

\section{Purpose of a systemic treatment of severe class II/1 cases}

The complete reduction of the overjet and the concomitant acquisition of mandibular kinematics that reflect a physiological masticatory function are, in our view, necessary and sufficient conditions for a stable a little trivial aphorism: chewing must work for us!

The modification of mandibular kinematics is the consequence of threedimensional anatomical changes and, in particular, of the change in orientation of the occlusal plane. Its reorientation transforms the masticatory cycles. Therefore, post-therapeutic mandibular kinematics, which is a reflection of this, should have a propulsive (physiological) 
component concerning the incisal blocks.

In practice, this is characterized by entries and exits of cycles relating the distal slope of the lower canine and the mesial edge of the upper canine. As for the lower incisors, they come end to end with the upper lateral incisor when the canines are, themselves, end to end. This functional rehabilitation of the anterior guide is a guarantee of the stability of the orthodontic correction because it prevents the recurrence of the overbite and, with it, the recurrence of the overjet and class II malocclusion.

\section{DEVICES USED FOR THE SYSTEMIC TREATMENT OF SEVERE CLASS II/1 CASES}

\section{Multi-attachment device}

The methods employed are less important than understanding the objectives to be achieved. They are modifiable as long as they fulfill the "specifications." Nevertheless, here we will confine ourselves to describing the tools that we use and that we know relatively well, namely the equipment used in the Tip Edge ${ }^{\complement}$ technique (but with a protocol different from that proposed by Parkhouse ${ }^{11}$ ):

- $\quad$ Tip-Edge Plus ${ }^{\circledR}$ attachments (low friction)

- double molar tubes: occlusal tube $(0.022 \times 0.028)$ and gingival tube (0.036)

- $\quad$ 0.016" Australian steel archwire

Other attachments can be used as long as they meet the required mechanical criteria, particularly low friction.

\section{Fixed anterior bite plate}

FABP is a mini-resin plate covering the upper retro-incisal area from canine to canine. It is fixed using a photopolymerizable adhesive on the occlusal surfaces of the upper premolars via a $0.032 "$ steel wire.
The thickness of the elevation causes total disocclusion of the lateral sectors. The inter-arch ratios, in those first days, are therefore reduced to the contacts between the lower incisors and the FABP resin (Figs. 2a, 2b, and 3).

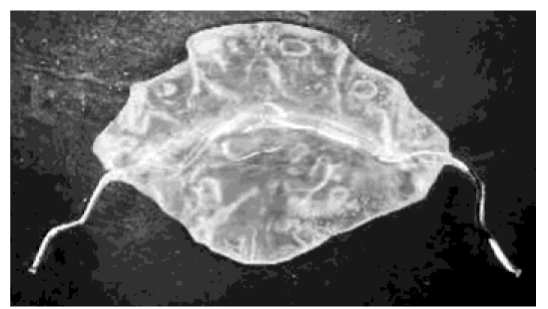

Figure $2 a$

Fixed anterior bite platediverging with the Camper plan in Class II.

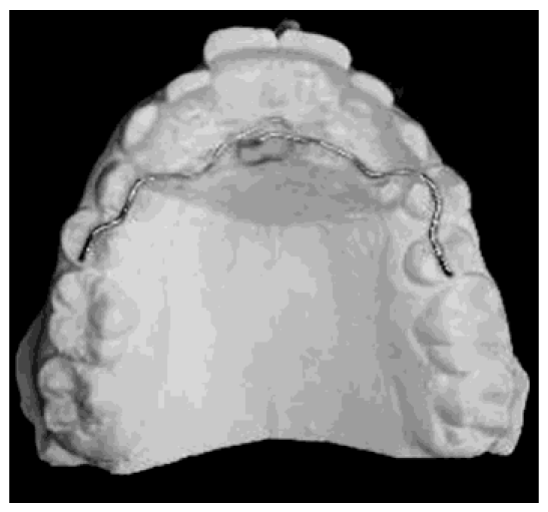

Figure $2 b$

Fixed anterior bite plate on molding 


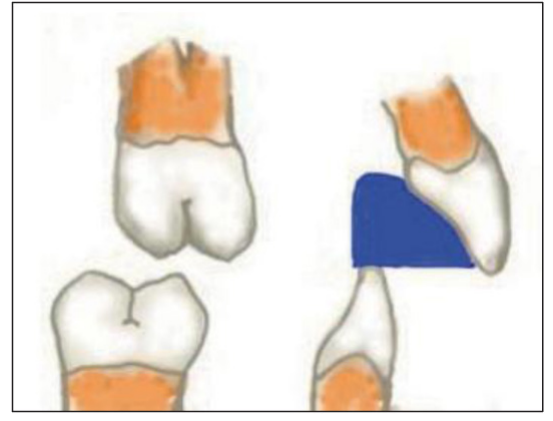

Figure 3

Major disocclusion caused by the placement of the fixed anterior bite plate (in blue).

Retro-incisive bite planes have already been used by various authors, either for problems such as bruxism1 or in the form of a retro-incisal plane on removable devices to correct earIy class $\mid 1,17,18$, and more rarely, with a multi-attachment device and, in this case, with the aim of avoiding interferences between the upper incisors and the bonded attachments of the lower incisors to reduce the risk of detaching the attachments.

Here, the role of the retro-incisal bite plane is different and very precise: the FABP, i.e., the retro-incisal plane, is fixed to the upper premolars to create a permanent posterior inocclusion space (for 3-4 months) (Fig. 3) allowing the eruption of upper molars to be induced by the tip back in the 0.016" upper arch.

The complete device is put in place as soon as possible, usually during the first 6 months of treatment (Fig. 4). The objective is to correct the overjet and overbite first and then to correct the associated anomalies (alignment of the teeth of the upper arch, torque, etc.)

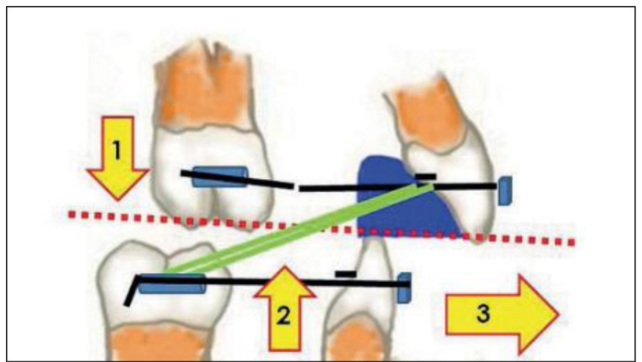

Figure 4

Complete device in place: in blue, the fixed anterior bite plate; in black, the0.016" Australian

steel archwire; in green, the class II light force elastics. The yellow arrows indicate the effects produced by the device: 1) Upper molar extrusion, 2) Lower leveling, 3) Mandibular advancement. These joint eff-ects lead to the reorientation of the occlusal plane.

\subsection{6" Australian steel archwire with molar tip-back at the upper arch}

An Australian steel archwire (preferably) is ligated on the upper four incisors (or sometimes from canine to canine), excluding premolars; we placed a $25^{\circ}$ molar tip-back 2 or $3 \mathrm{~mm}$ in front of the gingival tube of the molar braces (Fig. 4).

This tip-back will not cause ingression of the incisors but will cause regression of the upper molars because of the favorable force ratio to the latter (resistance of the periodontium and incisor roots versus initial disocclusion of 16 and 26 due to the presence of the FABP).

\section{Class II inter-arch elastics}

The action of inter-arch elastics is decisive in the correction of the overjet and the orientation of the occlusal 
plane. Their strength remains moderate: 3-4 oz (approximately 70-90 g). Elastic wearing must be strictly according to prescription:

- worn 23 h a day;

- replaced every 3 or $4 \mathrm{~h}$ throughout the day.

Patient cooperation is therefore essential to the good conduct and achievement of the treatment objectives. If these conditions are met, most of the overjet correction takes place over 3 or 4 months.

In addition, the action of the elastics contributes to the leveling of the lower arch. It is crucial for the success of the correction to insist on the following patient requirement: patients are expected to be completely cooperative because in terms of the effectiveness of the device, it's all or nothing! The wearing of elastics is inseparable from the action of the FABP.

\subsection{6" Australian steel arch wires to "square" the lower arch}

The shape of the lower arch is also important for the success of the method. The lower arch should be prepared beforehand, i.e., it should be given a "square shape" (Planas ${ }^{12}$ ) to keep the mandible in an advanced position after the correction of the overjet. This arch shape contributes to the acquisition of a functional anterior guide, i.e., friction between the lower and upper incisors essential for the durability of the correction.
Indeed, the correction of the overjet-and its stability-cannot be fully obtained if this condition is not fulfilled: if the shape of the mandibular arch is too "pointed," it will cause interference with the upper incisors during cycle entries, which causes a mandibular retrusion reflex and therefore instability of the orthodontic correction.

Let us recall here that the morphological changes must allow kinematics, whose propulsive component produces friction between the lower and upper incisors and not interference, as is the case when the lower arch is "pointed."

Therefore, whenever possible, the usual treatment protocol begins:

1. by shaping the lower arch with the multi-attachment device placed first at the lower arch (and not the upper arch);

2. by proceeding with the help of the 0.016" Australian steel archwire, shaped into a "square," after a preliminary "alignment and leveling." This requires us to shape the 0.016" steel archwire so that the lower canines are moved laterally to their functional position to allow for the correct inputs and outputs of the masticatory cycle. This repositioning of the lower canines (possibly by offsets for example) almost automatically results in the alignment of the lower incisal block in a pseudo-straight line, hence the term "square shape," an excessive but didactic term coined by P. Planas. 


\section{DESCRIPTION OF CLINICAL CASES ILLUSTRATING THE ABOVE}

\section{Olivia's case}

Born on $07 / 07 / 92$

Start of treatment: 04/18/05; End of treatment: 08/23/06; Duration of treatment: 16 months

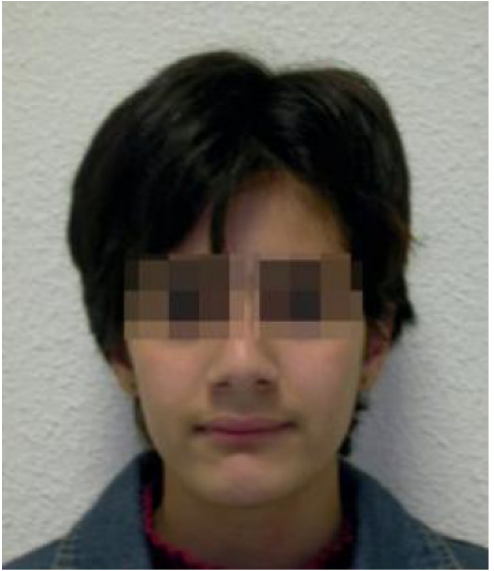

a

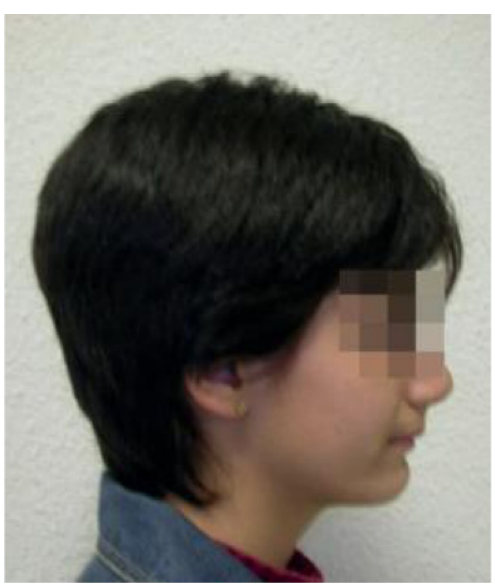

b

Figure 5

Olivia T., 01/03/05, at age 14: front (a) and side (b) views before treatment. The treatment was started on 04/18/05 and ended on 08/23/06, an exceptionally short duration of 16 months.

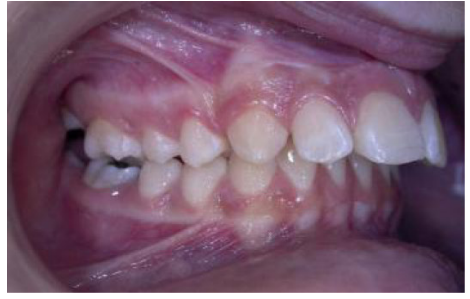

a

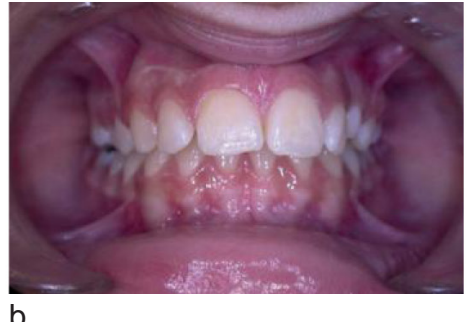

Figure 6

Intraoral views before treatment of maximal intercuspation in occlusion: right (a), front (b), and left (c) views.

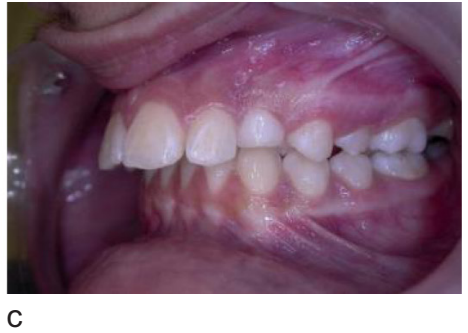

$\mathrm{C}$ 


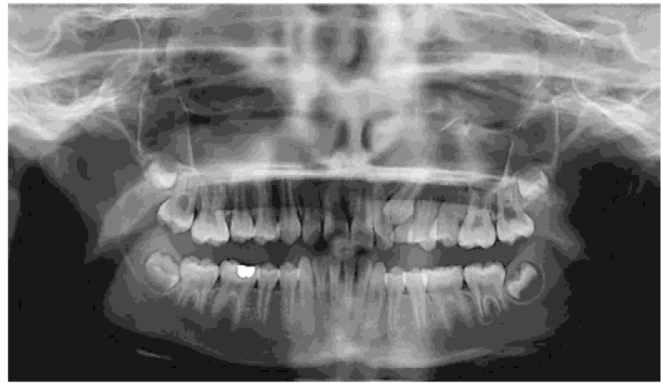

Figure 7

Panoramic X-ray before treatment.

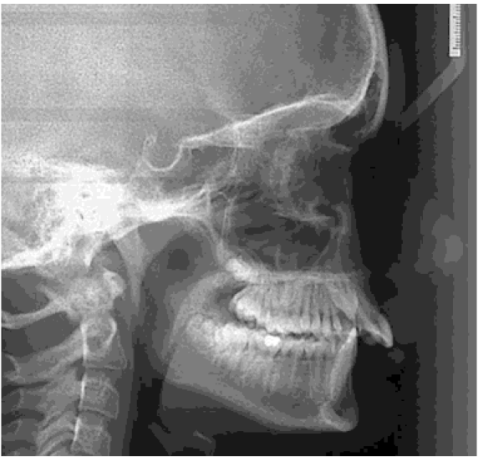

Figure 8

Profile teleradiography showing class II/1 with mandibular retrognathia (SNB 77\%, Wits: $5 \mathrm{~mm}$ ) on a highly hypodivergent skeletal pattern (FMA: $8^{\circ}$ ). There is also a large incidence of incisal proalveolia (I/F: 132\%). The overjet is $11 \mathrm{~mm}$.

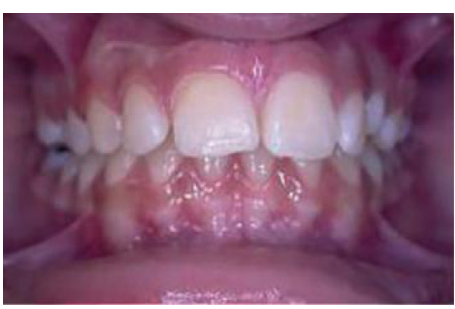

a

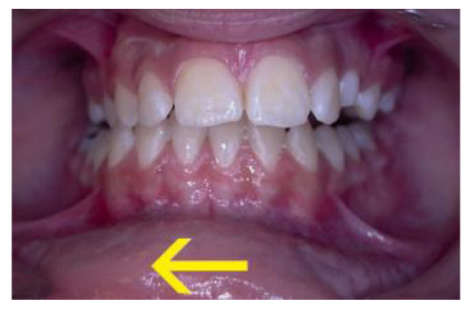

b

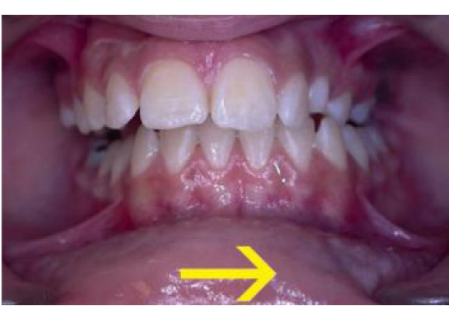

C

Figure 9

Intraoral views before treatment of maximal intercuspation in occlusion(a) and during mandibular lateral movements on the right (b) and left (c) showing frontal kinematics without a propulsive component.

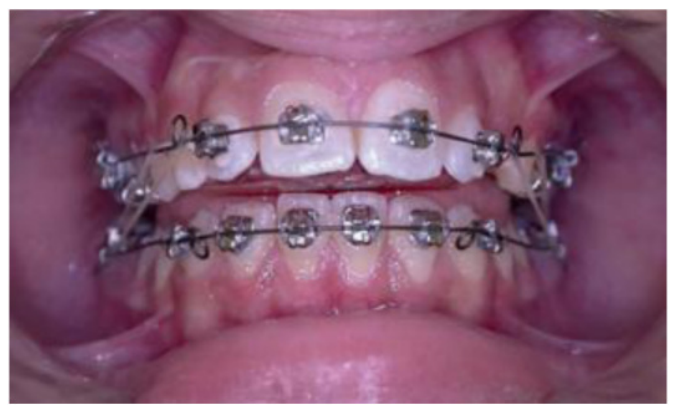

Figure 10

One month after the start of treatment (05/16/05), placement of the FABP and class II interarches elastic traction. The arches, upper and lower, are made of 0.016" Australian steel. 


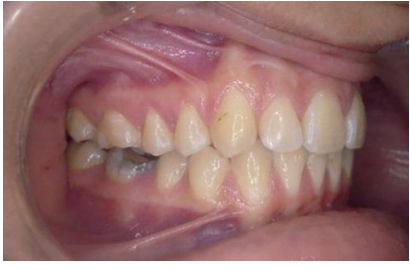

a

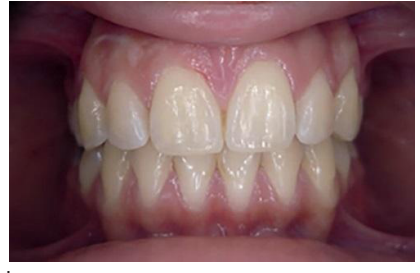

b

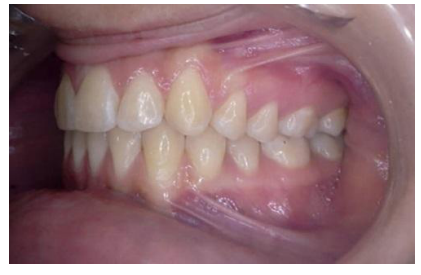

c

Figure 11

On 02/09/09, 2 years and 6 months after treatment, intraoral views of maximal intercuspation in occlusion on the right side (a), front (b), and left side (c).

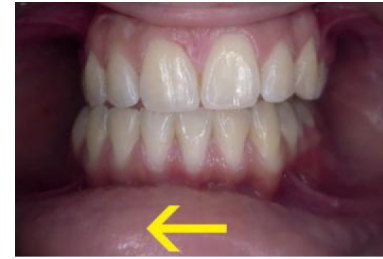

a

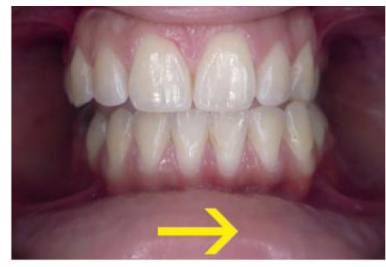

b

Figure 12

At the same time, intraoral views of mandibular lateral movements on the right (a) and left

(b) showing correct kinematics with a physiological propulsive component.

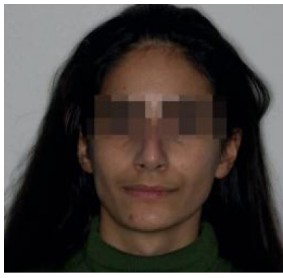

a

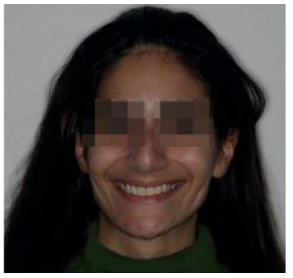

b

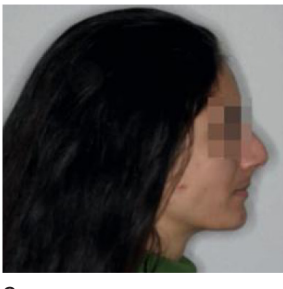

Figure 13

On February 15, 2017, 10 years and 7 months after treatment, front view (a), smile (b) and profile (c).

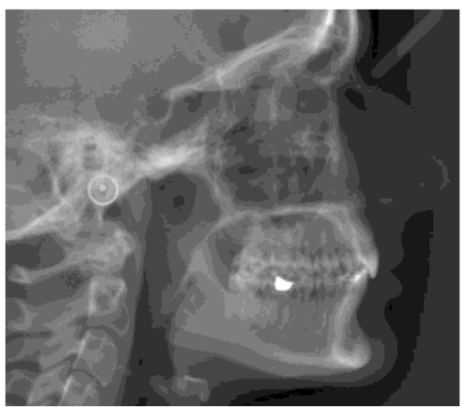

Figure 14

Teleradiography of profile more than 10 years after treatment showing skeletal and dental

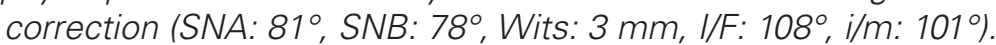



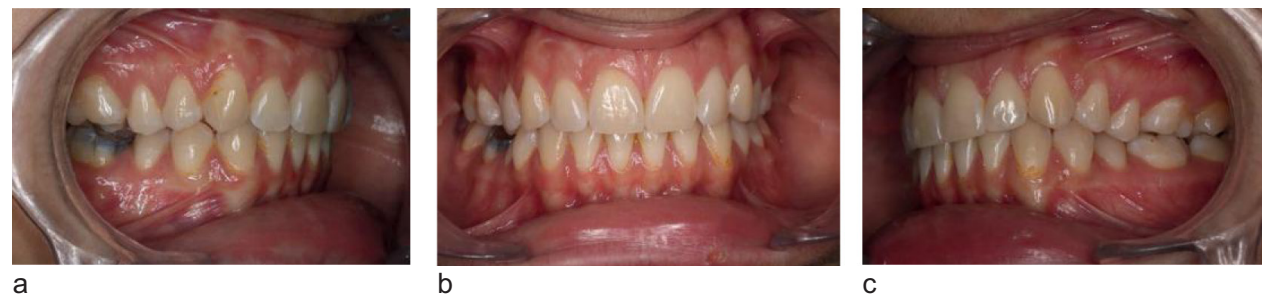

Figure 15

On 02/15/17, 10 years and 7 months after treatment, intraoral views of maximal intercuspation in occlusion on the right side (a), front (b), and left side (c).

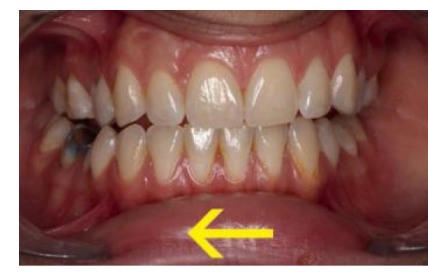

a

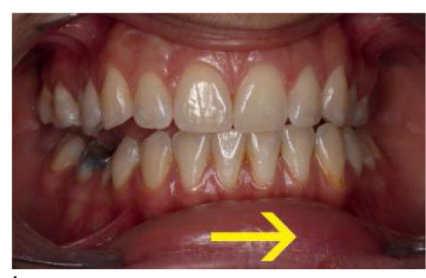

b

Figure 16

On 02/15/17, intraoral views during mandibular lateral movements on the right (a) and on the left (b) showing the correct kinematics with the morphological correction, which explains the stability of the correction.

\section{Case of Chloe P.}

Born on: 12/29/03

Start of treatment: 06/17/14 at the age of 10 years and 6 months; End of treatment: 07/18/16; Duration: 26 months

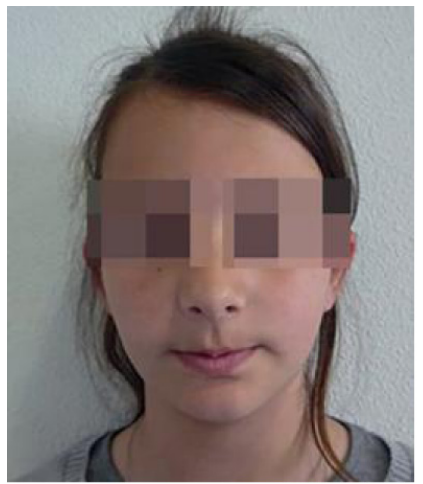

a

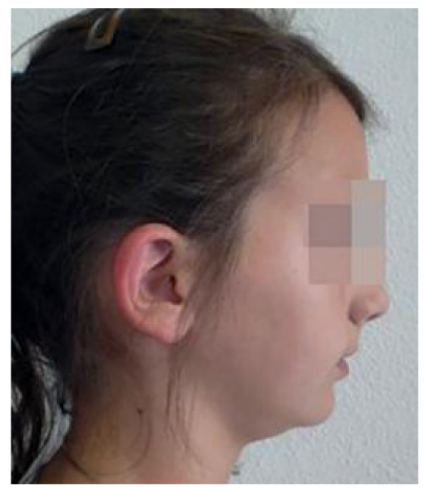

b

Figure 17

Front view (a) and profile (b) before treatment. 


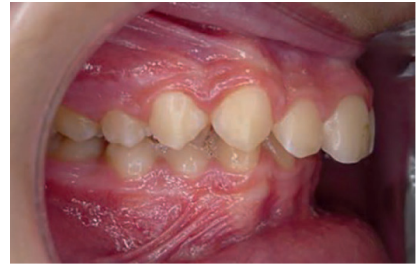

a

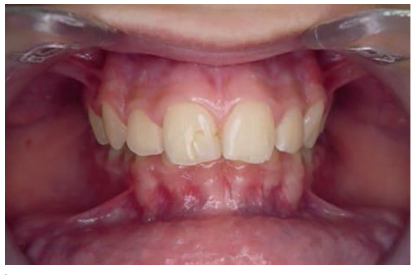

b

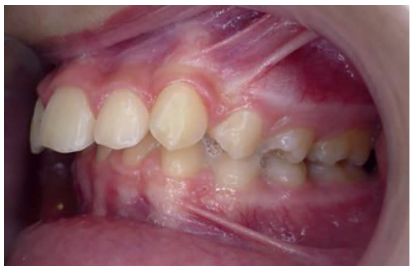

c

Figure 18

On 03/10/14, intraoral views before treatment of maximal intercuspation in occlusion on the right side (a), front (b), and left side (c) showing class II/1 molar and canine and an overjet of $10 \mathrm{~mm}$.

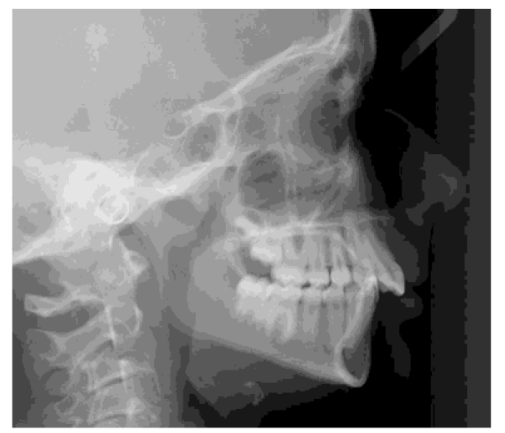

Figure 19

Teleradiography of profile showing mandibular retrognathia (SNB: $72^{\circ}$, Wits $4.5 \mathrm{~mm}$ ), a slight incisal pro-version (I/F: $\left.114^{\circ}\right)$, incisors (i/m: $\left.87^{\circ}\right)$, and the overjet of $10 \mathrm{~mm}$ on a normodivergent structure (FMA: $24 \%$.

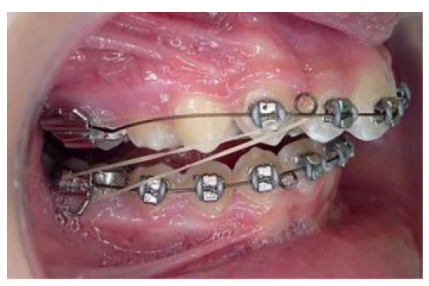

a

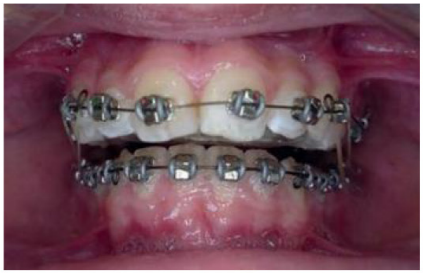

b

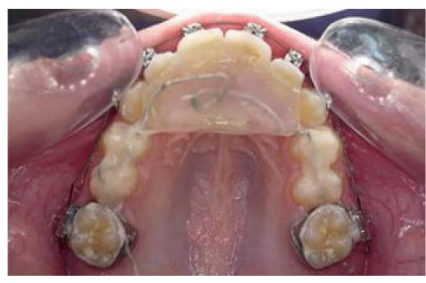

d

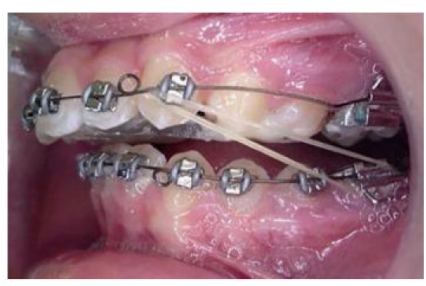

C

Figure 20

On 07/15/14, one month after the start of treatment, intraoral views showing, in occlusal view, (d) the FABP in place and the class I/ inter-arch light elastic traction (a, c ), and the maximal intercuspation in occlusion from the front (b). 


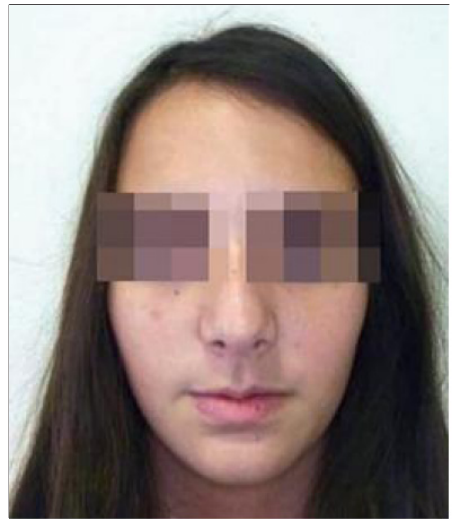

a

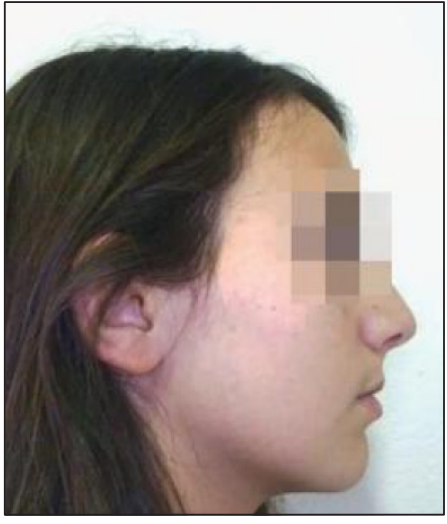

b

Figure 21

On 01/18/17, six months after treatment, front view (a) and profile (b).

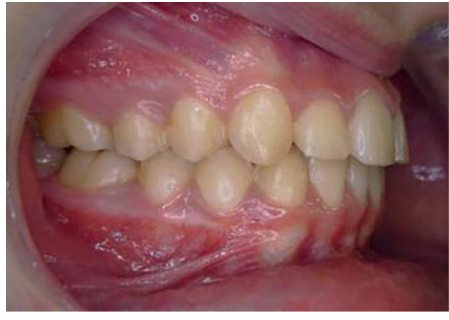

a

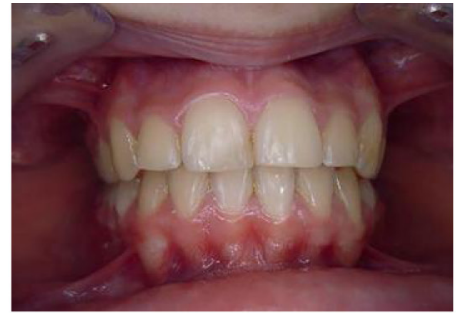

b

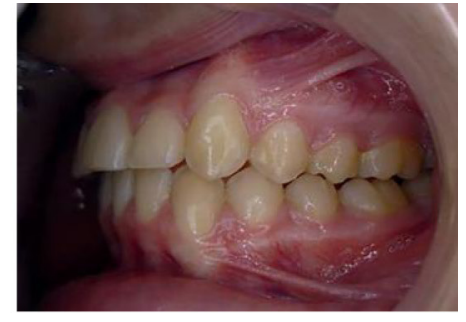

C

Figure 22

Intraoral views 6 months after treatment of maximal intercuspation in occlusion on the right (a), front (b), and left (c) showing correction of occlusion.

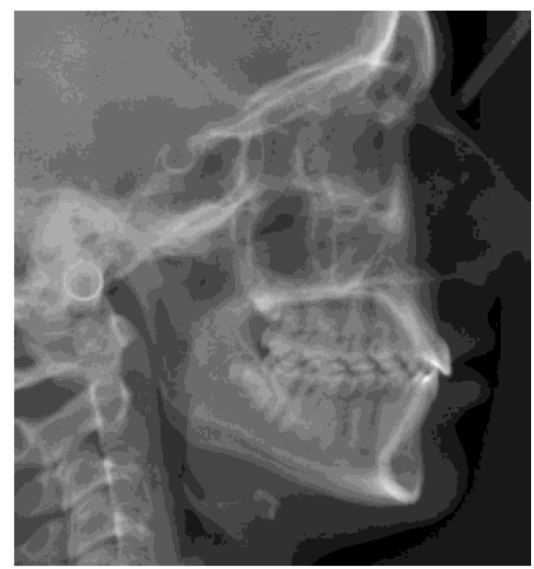

Figure 23

Teleradiography in profile on 05/17/17, i.e., 10 months after the treatment showing the skeletal correction (SNA: 78 ${ }^{\circ}$ SNB: 76', Wits: $0 \mathrm{~mm}$ ). The upper incisors have the same axis (I/F: $\left.113^{\circ}\right)$ while the lower incisors have a slightly lower angle (i/m: $\left.96^{\circ}\right)$. 


\section{CONCLUSION}

The systemic approach to orthodontics has led to the development of a treatment protocol to correct severe class II/1 by introducing a radical change in masticatory cycles, within a relatively reasonable time frame $(2.5$ years on average), without the need for orthognathic surgery. Of course, successful treatment is only possible if the adolescent is motivated and cooperates by rigorously wearing class II inter-arch elastics.
Morphological correction is stable in the long term because the threedimensional shape of the arches-and in particular their spatial orientationis compatible with an effective physiological mastication 12,14,19, which can ultimately be assimilated to a natural restraint device.

Conflict of interest: The authors declare no conflicts of interest.

\section{BIBLIOGRAPHY}

1. Bortoletto CC. et al. Evaluation of Cranio-cervical Posture in Children with Bruxism Before and After Bite Plate Therapy: A Pilot Project. J Phys Ther Sci 2014;26:1125-1128.

2. Delaire J, Salagnac JM, Notari J. Diagnostic des dysmorphoses dento-maxilo-faciales. Apports de I'analyse architecturale informatisée. Actual Odontostomatol (Paris) 1994;187:479-511.

3. Gaspard M. Acquisition et exercice de la fonction masticatrice chez l'enfant et l'adolescent. Revue Orthop Dento-faciale 2001;35:349-403.

4. Hun-Mu Yang, Jung-Yul Cha, Ki-Seok Hong, Jong-Tae Park. Three-dimensional finite element analysis of unilateral mastication in malocclusion cases using conebeam computed tomography and a motion capture system. J Periodontal Implant Sci2016;46(2):96-106.

5. Katsaros C, Zissis A, Bressin A, Kiliaridis S. Functional influence on sutural bone apposition in the growing rat. Am J of Orthod Dentofacial Othop 2006;129:352-357.

6. Kiliaridis S, Engström C, Lindskog-Stokland B, Katsaros C. Craniofacial bone remodeling in growing rats fed a low calcium and vitamin $D$ deficient diet and the influence of mastication function. Acta Odontol Scand 1996;54(5):HS 320-326.

7. Kolf J. Les classes II division 1. Historique et évolution des concepts. EMC - Médecine buccale 2008;3(1):1-20 [Article 28-620-M-10].

8. Lagaida M, White GE. Unilateral mastication and facial formation. J Pedod 1983:7:127-134.

9. Lauret JF, Le Gall M. La mastication, une réalité oubliée par l'occlusodontologie ? Les Cahiers de prothèse 1994:85.

10. Mavropoulos S, Kiliaridis S, Bresin A, Ammaw P. Effect of different masticatory functionnal and mechanical demands on the structural adaptation of the mandibular alveolar bone in young growing rats. Bone 2004;35, sup:191-197.

11. Parkhouse R. Tip-Edge Orthodontics and the Plus Bracket, 2nd Edition. Mosby Ltd, 2009.

12. Planas P. La Réhabilitation Neuro-Occlusale (RNO). Traduction Chateau M, Kolf J, $2^{2}$ édition. Paris : CDP, 2007. 
13. Raymond JL. Traitement orthopédique des classes III : approche occlusale et fonctionnelle $2^{e}$ édition. Ed. Empresa, 2011.

14. Raymond JL, Kolf J. Complexité du système masticateur. Manifeste pour un nouveau paradigme de l'occlusion dentaire. Ed. Empresa, 2014.

15. Salagnac JM. Classes II division 2. Identification, classification des différentes variétés cliniques à l'aide de l'analyse architecturale et structurale craniofaciale de J. Delaire. Déductions thérapeutiques. Orthod Fr 1982;53:623-640.

16. Sato $H$, Kawamura A, Yamaguchi M. Relationship between masticatoty function and internal structure of the mandibule based on computed tomography findings. Am Ass of Orthod 2005: 766-773.

17. Showkatbakhsh R, Meybodi SE, Jamilian S, Meybodi ARF, Meybodi EM. Treatment effects of R-appliance and Anterior Inclined Bite Plate in Class II, Division I malocclusion. J Appl Oral Sci 2011;19(6):634-638.

18. Veerasamy K. Le plan rétro-incisif incliné dans le traitement de la rétrognathie mandibulaire. Mémoire CECSMO, Faculté de Lille, 2008.

19. Woda A, Fontenelle A. Physiologie de l'appareil manducateur. In : Château, Orthop Dento-Faciale Tome 1, Bases scientifiques 167-221. Paris: Ed. CDP,1993. 